\title{
Forced Oscillation Wind Tunnel Testing for FASER Flight Research Aircraft
}

\author{
Garrison Hoe ${ }^{*}$ \\ The Boeing Company, Seattle, WA, 98124 \\ D. Bruce Owens ${ }^{\dagger}$ \\ NASA Langley Research Center, Hampton, VA, 23681 \\ and \\ Casey Denham \\ Massachusetts Institute of Technology, Cambridge, MA, 02139
}

\begin{abstract}
As unmanned air vehicles (UAVs) continue to expand their flight envelopes into areas of high angular rate and high angle of attack, modeling the complex unsteady aerodynamics for simulation in these regimes has become more difficult using traditional methods. The goal of this experiment was to improve the current six degree-of-freedom aerodynamic model of a small UAV by replacing the analytically derived damping derivatives with experimentally derived values. The UAV is named the Free-flying Aircraft for Sub-scale Experimental Research, FASER, and was tested in the NASA Langley Research Center 12Foot Low-Speed Tunnel. The forced oscillation wind tunnel test technique was used to measure damping in the roll and yaw axes. By imparting a variety of sinusoidal motions, the effects of non-dimensional angular rate and reduced frequency were examined over a large range of angle of attack and side-slip combinations. Tests were performed at angles of attack from -5 to 40 degrees, sideslip angles of $-\mathbf{3 0}$ to 30 degrees, oscillation amplitudes from 5 to 30 degrees, and reduced frequencies from 0.010 to 0.133 . Additionally, the effect of aileron or elevator deflection on the damping coefficients was examined. Comparisons are made of two different data reduction methods used to obtain the damping derivatives. The results show that the damping derivatives are mainly a function of angle of attack and have dependence on the non-dimensional rate and reduced frequency only in the stall/post-stall regime.
\end{abstract}

\section{Nomenclature}

$b \quad=$ wing span, $\mathrm{ft}$

c.g. $\quad=$ center of gravity

$C_{L} \quad=$ lift coefficient

$C_{l} \quad=$ rolling moment coefficient

$C_{m} \quad=$ pitching moment coefficient

$C_{n} \quad=$ yawing moment coefficient

$C_{l p} \quad=$ damping-in-roll derivative due to roll rate, per radian

$C_{l r} \quad=$ damping-in-roll derivative due to yaw rate, per radian

\footnotetext{
${ }^{*}$ Propulsion Systems Engineer, Airplane Maintenance Engineering, MC 2J-02.

${ }^{\dagger}$ Senior Engineer, Flight Dynamics Branch, M/S 308, Associate Fellow.

* Student, MIT.
} 


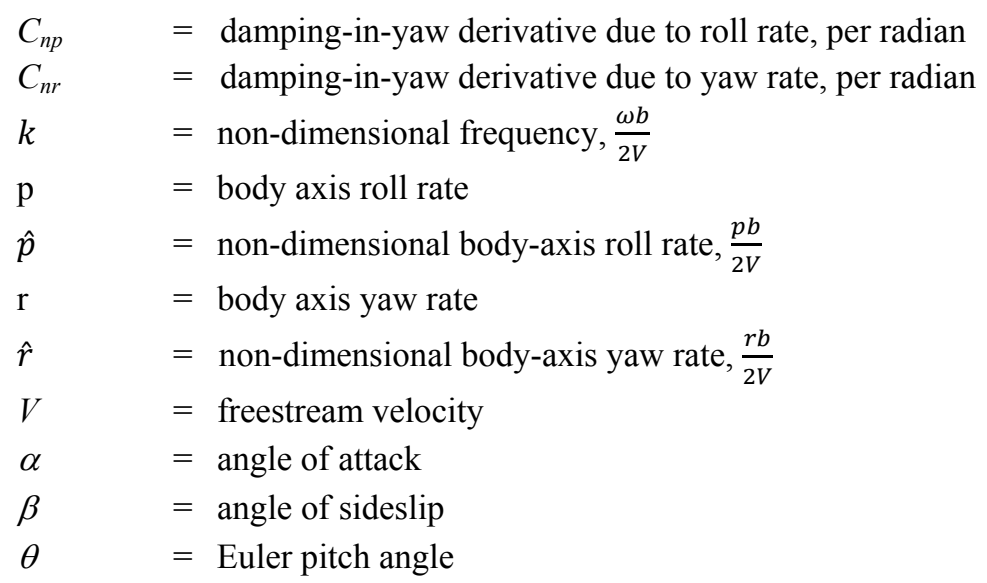

\section{Introduction}

$\mathrm{H}$ AVING an accurate prediction of airplane aerodynamic characteristics is required to develop a high fidelity model and to design robust controllers. Most aerodynamic models are sufficient in representing the static aerodynamics but can be deficient in the modeling of the nonlinear viscous damping effects especially at high angles of attack and sideslip. The aerodynamic derivatives found from static wind tunnel testing will predict the dominant characteristics of the aircraft motion about trim conditions at low angle of attack, while the damping derivatives are requisite for accurately describing maneuvering flight, especially at stall and post-stall angles of attack. Dynamic

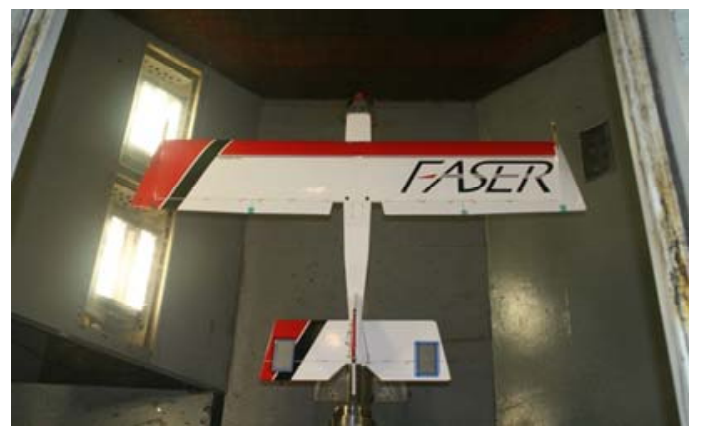

Figure 1. FASER mounted in the NASA LaRC 12-FT Low-Speed Tunnel. wind tunnel test techniques are utilized to characterize the damping effects. The test aircraft, FASER (Fig. 1), has been the subject of a number of wind tunnel and flight tests and has been the subject of many other research projects $^{1-5}$. The impetus for FASER was a low-cost, low-maintenance platform to be used to develop or improve test techniques. The same aircraft is used both in the wind tunnel and in flight so that outer mold line and scaling effects are minimized. Since FASER has a conventional planform with well-known low angle of attack characteristics it is expected that the damping derivatives calculated from experimental data in this range will agree with analytical predictions. A goal of the experiment was to determine the high angle of attack damping derivative values as these were expected to deviate significantly from the analytical predictions. These experimental values can then be used in simulation comparisons to better understand the level of fidelity required for the damping derivatives.

NASA LaRC has utilized dynamic testing methods to characterize the flight dynamics of many aerospace vehicles in the past ${ }^{6}$. Forced oscillation testing is a type of dynamic testing technique that was used in this experiment. It is known from these prior tests that the damping derivatives can be functions of non-dimensional rate and reduced frequency parameter, especially in the stall/post-stall regime. Sinusoidal, one-degree of freedom oscillations were performed about the roll and yaw axes, and time history data of the aerodynamic force and moment coefficients were recorded. Although not a requisite, sinusoidal motion is used because aircraft dynamics are, in general, described by second-order harmonics. Similitude requirements dictate that ground tests must have the same non-dimensional angular rate and reduced frequency parameter as in flight. As mentioned, the same aircraft is used for flight and ground testing, therefore making these requirements more achievable compared to a sub-scale model test. For sub-scale models the similitude requirements are normally met by oscillating at higher frequencies than observed in flight. This places additional burden on the forced oscillation mechanism. The other way is for the test to be conducted at lower freestream velocities than in flight, further reducing the already typically low Reynolds number. 
The apparatus and methods used to conduct the test are presented first. This section includes details on the geometry and wind tunnel model support system interference considerations. Also, the section includes a discussion on the data reduction methods and an overview of the static aerodynamics. The section concludes with the test matrix requirements. Following the apparatus and methods section, the results of the test are discussed. The paper concludes with a summary and future work.

\section{Apparatus and Methods}

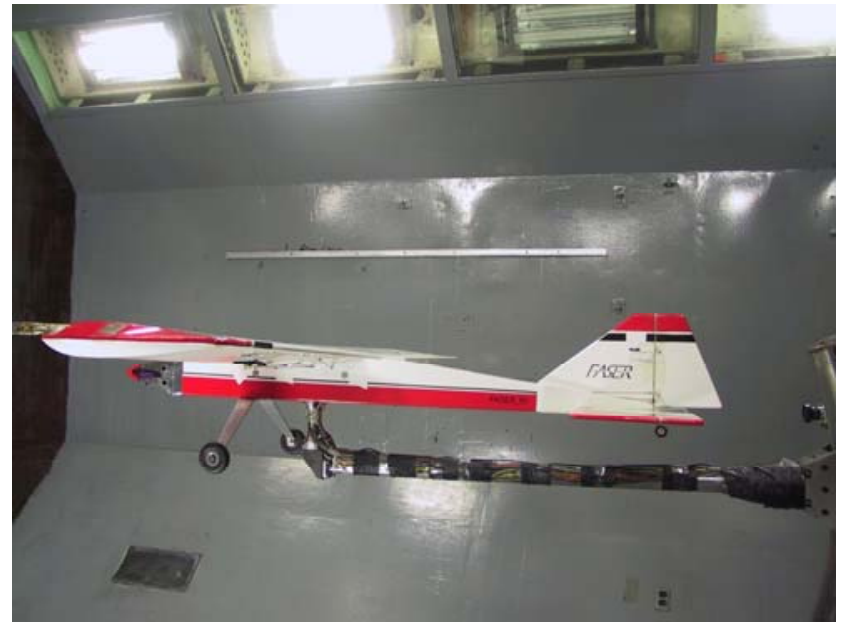

Figure 2. FASER mounted for static force and moment tests prior to the current forced oscillation.

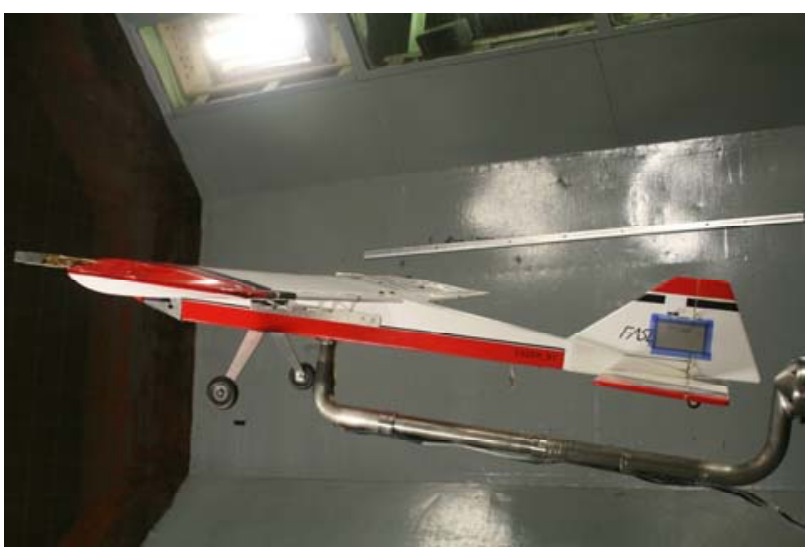

Figure 3. FASER mounted for roll oscillation runs.

sets can be made without the need to correct for tunnel-totunnel variances. Prior tests have measured static forces and moments, control power derivatives, thrust effects, and air data probe installation effects. As stated earlier, the damping derivatives are determined experimentally using the forced oscillation technique $^{6}$. This tunnel was recently updated with a new forced oscillation apparatus that allows for arbitrary input functions to create desired dynamic motions. Details of the apparatus can be found in reference 6 . The mounting for forced oscillation testing requires a different sting than the one used for static testing (Fig. 2). Additionally, the sting
Table 1. Summary of Geometric Characteristics

\begin{tabular}{|l|l|}
\hline Wing Span, $\mathrm{ft}$ & 6.29 \\
\hline Reference Wing Area, $\mathrm{ft}^{2}$ & 8.28 \\
\hline Aspect Ratio & 4.42 \\
\hline Mean Aerodynamic Chord, $\mathrm{ft}$ & 1.42 \\
\hline
\end{tabular}

In this experiment the $6.3 \mathrm{ft}$ wingspan FASER UAV aircraft undergoes forced oscillation testing in the roll and yaw axis. FASER is a full scale test article with the same model being used both for flight tests and ground tests. The airframe is a Hanger 9 ARF Ultra-Stick ${ }^{\mathrm{TM}} 120$ kit-built tail-dragger. Key configuration details are specified in Table 1. The forced oscillation tests were conducted in the NASA Langley 12-FT Low Speed Tunnel (12-FT LST). All FASER wind tunnel tests have been conducted in the 12-FT LST. Therefore, assimilating the various data

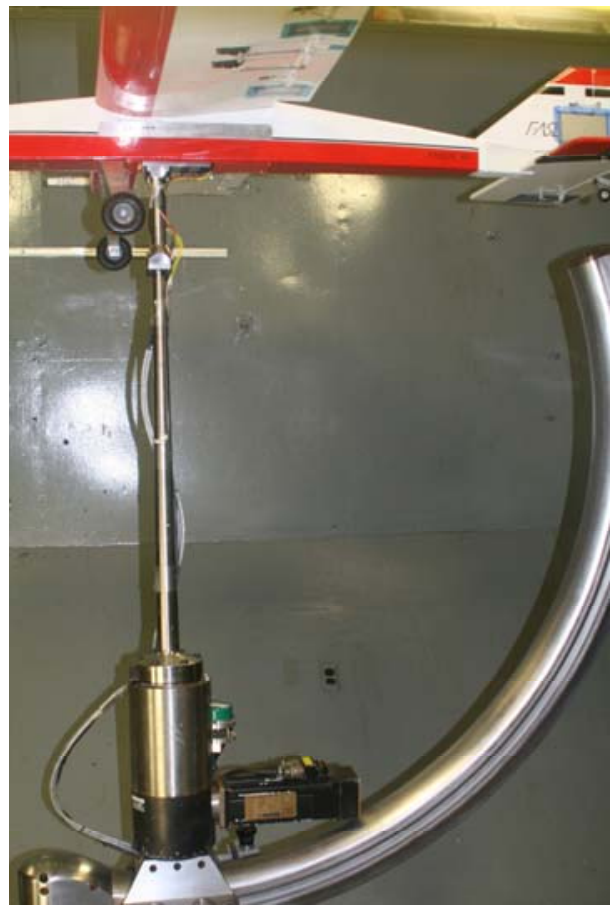

Figure 4. FASER mounted for yaw oscillation runs with the wings parallel to the test section floor. 


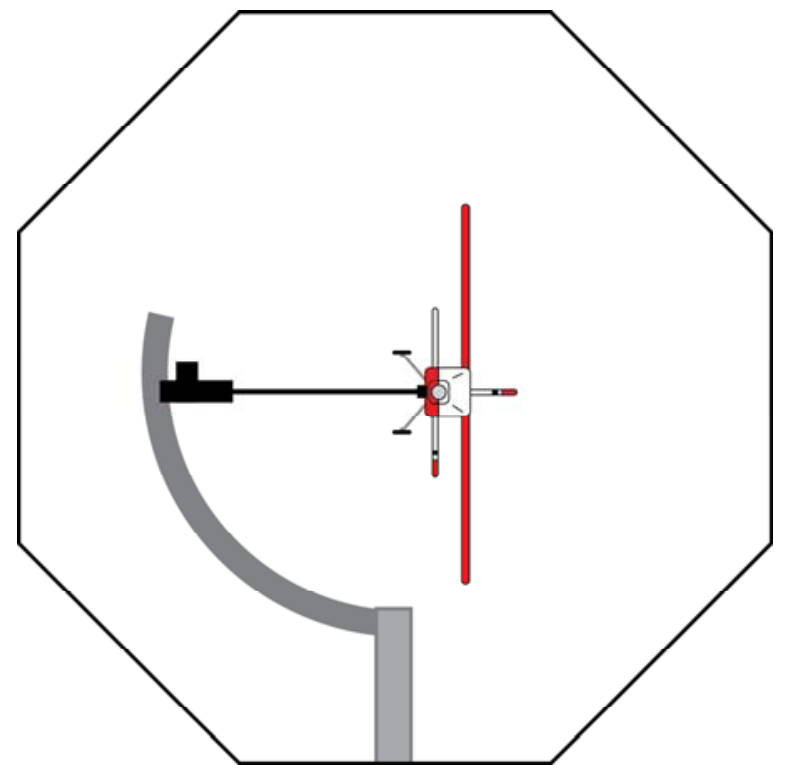

Figure 5. FASER mounted for yaw oscillation with the wings vertical. Nominal arrangement for yaw oscillation.

Fig. 7. These results are given for reference to the reader for discussion during the results and discussion section. Summarizing, Fig. 7 shows that the aerodynamics are linear up to $\alpha=13^{\circ}$ where wing stall begins. The pitching moment data shows that the model is statically stable through the stall angle-ofattack, having a stable break at the stall. The rolling and yawing moment curves in Fig. 7 show that at stall there is significant rolling and yawing moment asymmetry in the $\alpha=12^{\circ}$ to $20^{\circ}$ range. installation is different between roll damping (Fig. 3) and yaw damping (Figs. 4 and 5). Therefore, the different sting installations may cause different sting interference effects. In order to verify this, initial runs made in the test were static. The effect of these four different sting arrangements is shown in Fig. 6.

It can be seen that for $C_{L}$ vs $\alpha$ there is good agreement in the lower angles of attack range between the various mounting schemes. The discrepancy approaching stall is likely caused by the flow disturbance around the sting as it begins to interact with the flow over the wings. Note that when the aircraft was mounted upright (wings parallel to test section floor) for yaw oscillation that the angle of attack range is only $-5^{\circ}$ to $0^{\circ}$. The data for this mounting arrangement is shown in blue with diamond symbols. Since the main contributor to roll damping is the wing aerodynamics the similarity of the static and forced oscillation stings should not alter the wing flow field significantly.

The static aerodynamic characteristics are shown in

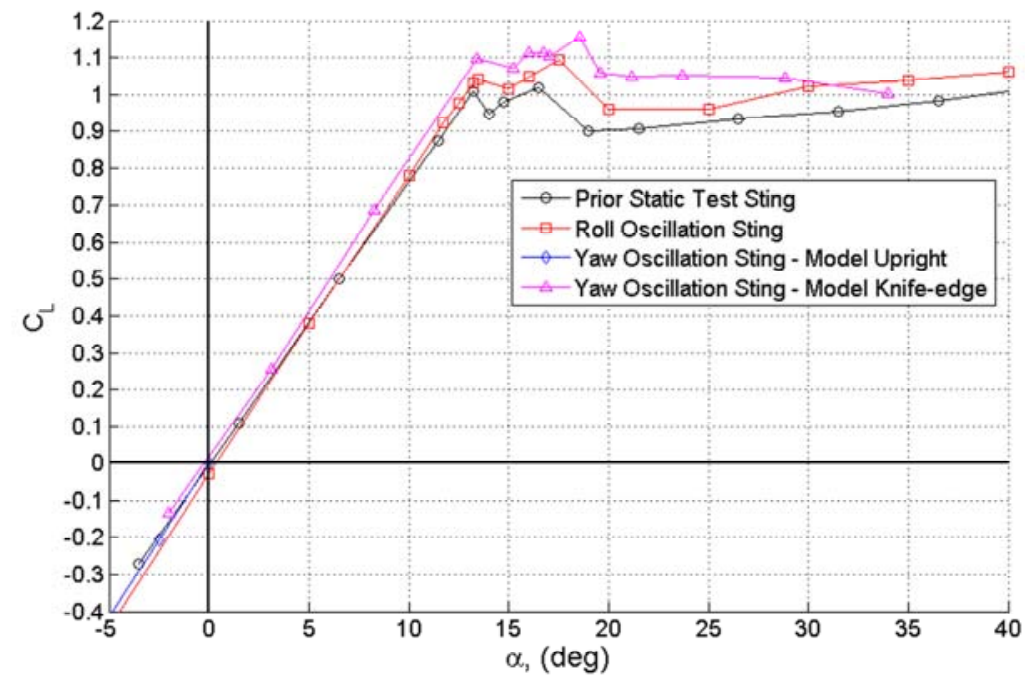

Figure 6. Effect of sting arrangement on lift characteristics. 

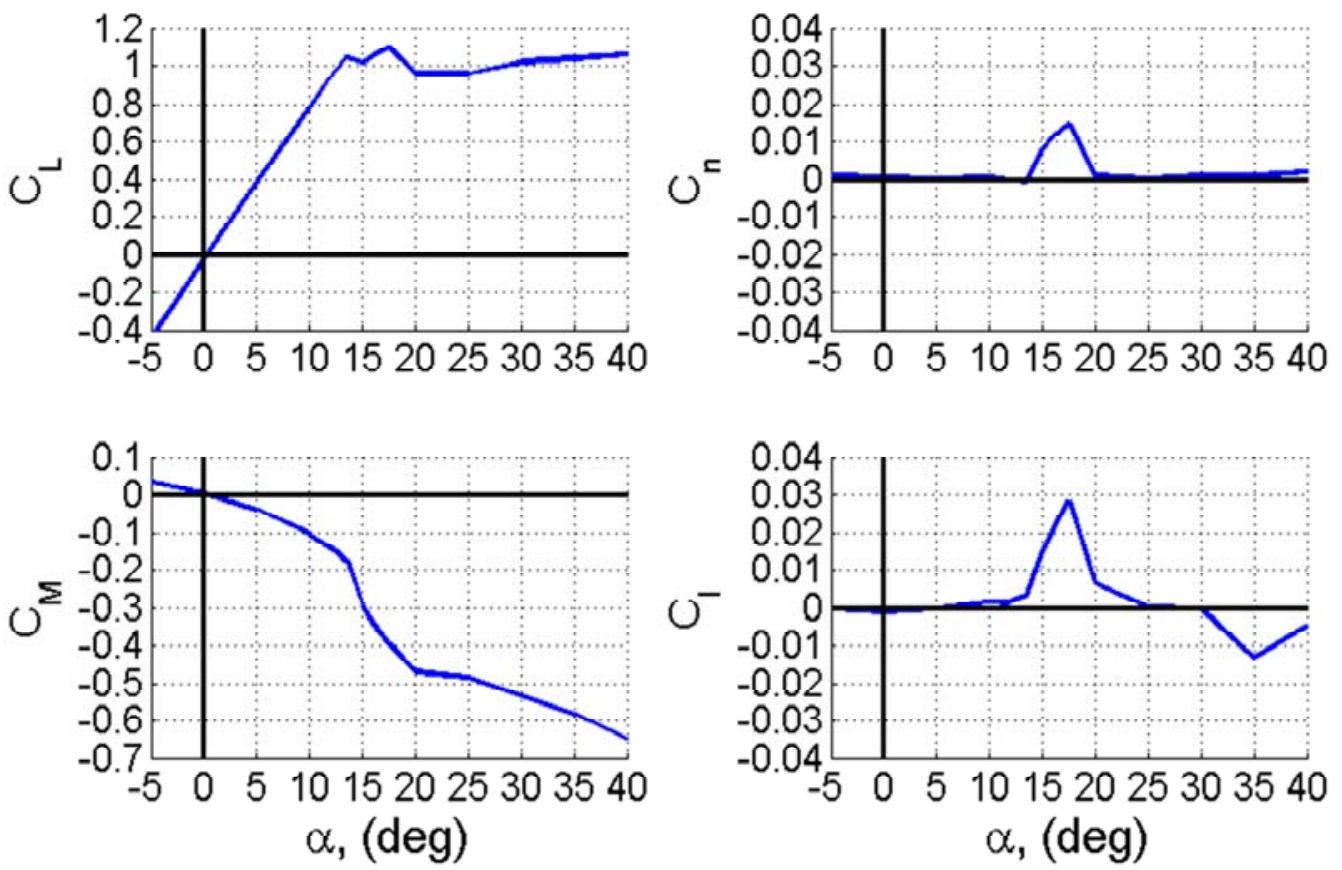

Figure 7. Summary of static aerodynamics. All control surfaces $=0^{\circ}$ and $\beta=0^{\circ}$.

\section{Data Reduction Methods}

Two methods have been used to derive the dynamic derivatives from the time histories of the moment coefficients. The first is called the integral method where the aerodynamic coefficients, called in-phase and out-ofphase components, are estimated by using measurement time histories. The in-phase component contains a static derivative and an oscillatory derivative, while the out-of-phase component is used to determine the damping derivative (Table 2). For details on the integral method see Ref. 7.
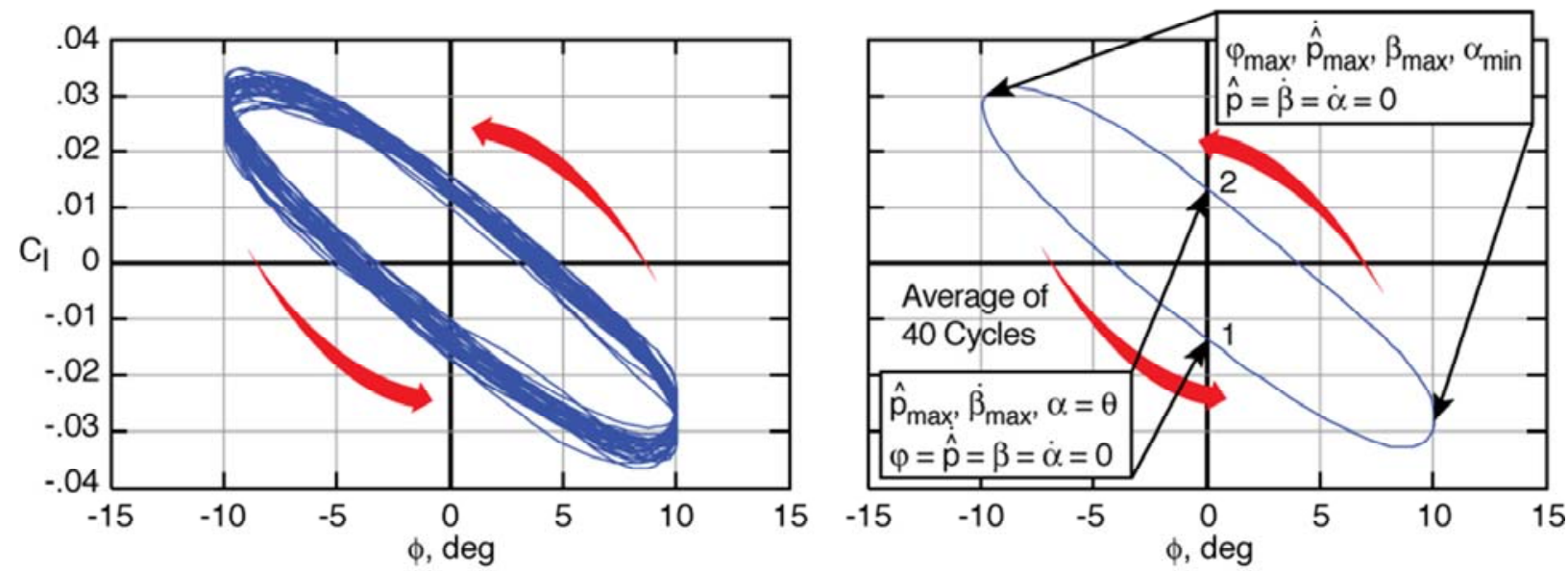

Figure 8. Time history of rolling moment coefficient verses roll angle for 40 continuous cycles (left) and the average cycle (right). (Example data not from FASER wind tunnel test used only for illustrative purposes.)

Another method is the single-point method which utilizes the time history data in the form of hysteresis loops of the balance moment data for each point in a run (Fig. 8). Oscillation data are recorded about the nominal $\alpha$ or $\beta$ where the acceleration is theoretically zero and non-dimensional rate is at a maximum for a preset number of 
Table 2 Primary averaged in-phase and out-of-phase body axes moment coefficients

\begin{tabular}{|c|l|l|}
\hline Oscillation axis & \multicolumn{1}{|c|}{ In-phase } & Out-of-phase (damping) \\
\hline Pitch & $\overline{C_{m_{e}}}=C_{m_{\alpha}}-k^{2} C_{m_{\dot{q}}}$ & $\overline{C_{m_{q}}}=C_{m_{q}}+C_{m_{\dot{\alpha}}}$ \\
\hline Roll & $\overline{C_{l_{\beta}}}=C_{l_{\beta}} \sin (\alpha)-k^{2} C_{l_{\dot{p}}}$ & $\overline{C_{l_{p}}}=C_{l_{p}}+C_{l_{\dot{\beta}}} \sin (\alpha)$ \\
\hline Yaw & $\overline{C_{n_{\beta}}}=C_{n_{\beta}} \cos (\alpha)+k^{2} C_{n_{\dot{r}}}$ & $\overline{C_{n_{r}}}=C_{n_{r}}-C_{n_{\dot{\beta}}} \cos (\alpha)$ \\
\hline
\end{tabular}

oscillations. The cycles of data are averaged to obtain a mean cycle. Using the mean cycle, the damping derivative value is then determined by subtracting the moment coefficient at the maximum negative rate from the maximum positive rate and dividing by the difference of these two rates

(Eq. 1). Compared to the integral method the single point method is a better representation of an instantaneous value of the derivative.

$$
\overline{C_{l p}}=\frac{C_{l_{2}}-C_{l_{1}}}{\hat{p}_{2}-\hat{p}_{1}}
$$

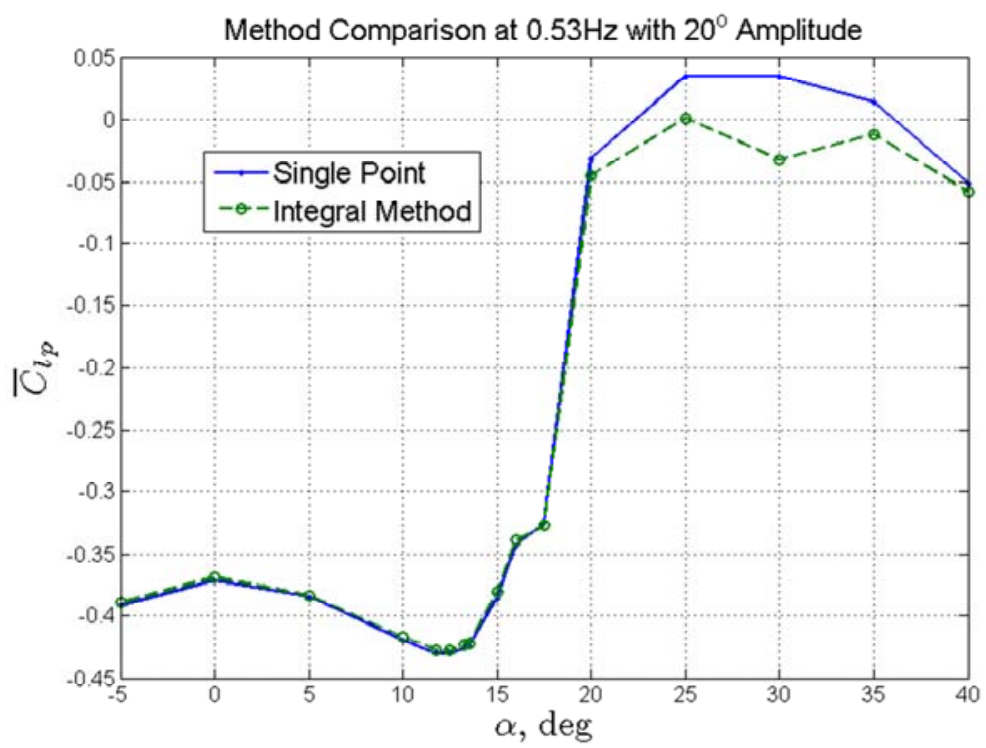

Figure 9. Comparison of the rolling damping derivative computed from the integral method to the one computed from the single point method.

An example of a comparison between the integral and single point methods are shown in Fig. 9 of roll damping verses angle of attack. Effectively the integral method determines the average damping over the mean cycle. The integral method computes Fourier coefficients for the first harmonic that can be directly related to the in-phase and out-of-phase coefficients ${ }^{8}$ whereas the single point method is a linearization of the moment due to rate at the nominal $\alpha$ or $\beta$. A possible reason for the differences at high angle of attack is that the rolling moment coefficient hysteresis loops (Fig. 10) deviate significantly from an ellipse. The single point and integral methods approach the same value as the hysteresis loops approach an ellipse as seen for $\alpha=0^{\circ}$. Simulation aerodynamic models assume instantaneous values so the single point method is used to arrive at the dynamic damping derivatives. 

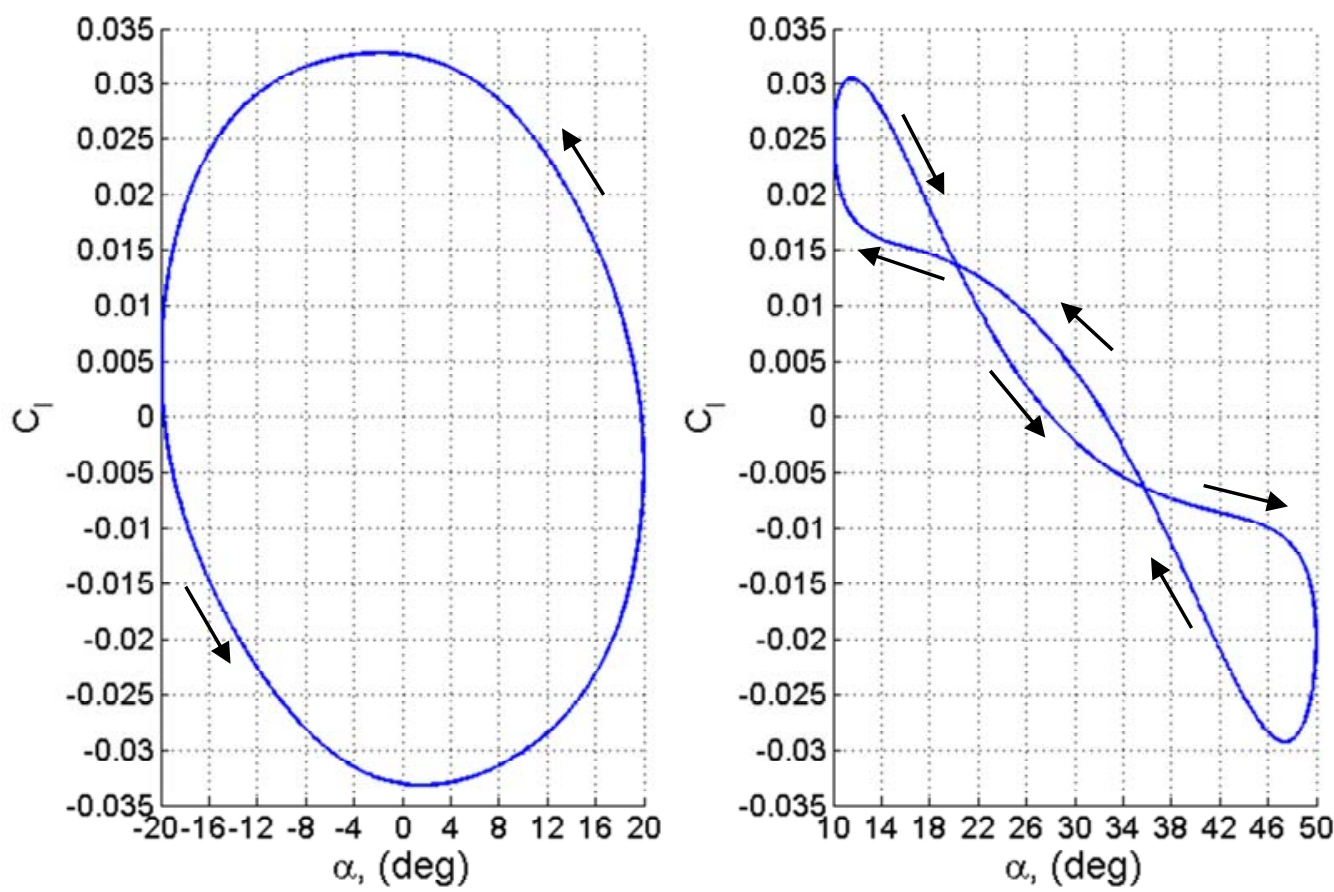

Figure 10. Rolling moment coefficient hysteresis loops for $\alpha=0^{\circ}$ and $\alpha=30^{\circ}$.

\section{Test Matrix Requirements}

The damping derivatives are measured at non-dimensional rate and reduced frequency parameters based on similitude requirements. This was achieved by examining unpublished flight test data collected from a $60 \%$ geometrically scaled UltraStick 25 flown at the University of Minnesota because of the large quantity of flight data and ease of access to the data. Reviewing the flight data yielded non-dimensional roll rates between $\hat{p}= \pm 0.1$ for most flights while some flights pushed it to $\hat{p}= \pm 0.22$. By linearizing the current FASER simulation model around a trim cruising condition, a Dutch-roll frequency of $0.53 \mathrm{~Hz}(k=0.255)$ was extracted and used as a nominal testing frequency. After determining the rate and frequency similitude values, the final range of rates and frequencies were chosen such that a parametric investigation could be conducted on rate and frequency effects. The upper bounds of these ranges were dictated by balance load limits. The $\alpha$ and $\beta$ sweeps values were chosen to match prior static wind tunnel test matrices which also provided good coverage of the flight envelope. For FASER the typical flight envelope includes angle of attack from -10 to 30 degrees and side slip in the range of \pm 25 degrees. Table 3 lists the range of the test parameters. 
Table 3 - Test Matrix Envelope

\begin{tabular}{|c|c|c|}
\hline Parameter & Roll Axis & Yaw Axis \\
\hline Amplitude of Oscillation, deg. & $5^{\circ}$ to $30^{\circ}$ & $5^{\circ}$ to $40^{\circ}$ \\
\hline Oscillation Frequency, Hz & $0.25 \rightarrow 1$ & $0.25 \rightarrow 1$ \\
\hline Reduced Frequency, $k$ & $0.12 \rightarrow 0.481$ & $0.12 \rightarrow 0.481$ \\
\hline Dynamic Pressure, psf & 2 & 2 \\
\hline Angle of Attack, deg. & $-5^{\circ} \rightarrow 40^{\circ}$ & $-5^{\circ} \rightarrow 30^{\circ}$ \\
\hline Sideslip, deg. & $-30^{\circ} \rightarrow 30^{\circ}$ & $-20^{\circ} \rightarrow 20^{\circ}$ \\
\hline$|\hat{p}|$ Maximum During Oscillation & $0.01 \rightarrow 0.098$ & N/A \\
\hline$|\hat{r}|$ Maximum During Oscillation & N/A & $0.01 \rightarrow 0.133$ \\
\hline
\end{tabular}

\section{Instrumentation}

To measure the aircraft forces and moments a NASA Langley internally-mounted six-component strain gauge balance was used. The position encoder signal from the forced oscillation rig was differentiated to calculate nondimensional angular rate. This angular rate was used in the data reduction. The strain gauge balance measured the weight, inertial moments (during forced oscillation), and aerodynamic forces and moments. Since the forced oscillation results in the balance reading the inertial moments associated with each frequency and amplitude a windoff tare with the model oscillating at the identical wind-on conditions must be conducted to remove these effects. It should be noted that there was no attempt to remove the effect of wind-off aerodynamic damping during these tare runs as the oscillation frequency and amplitudes were judge to be small enough to ignore the effect. All data were sampled at $250 \mathrm{~Hz}$ and an analog anti-aliasing filter in the data acquisition loop filters the signals at $50 \mathrm{~Hz}$.

\section{Results and Discussion}

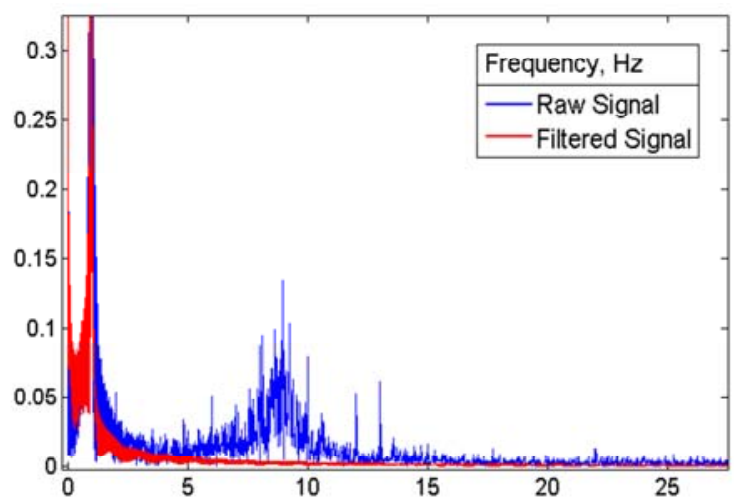

Figure 11. FFT plot showing signal analysis and noise filtering
Data for the roll axis will be presented first, followed by an overview of the yaw results. To reduce signal noise the data were digitally filtered based on examination of an FFT (Fast Fourier Transform) of the signal. Figure 11 shows an FFT of the unfiltered signal as well as the signal after applying a Butterworth filter with a cut-off frequency four times the oscillation frequency. As shown in the figure the signal information at the oscillation frequency of $1 \mathrm{~Hz}$ is maintained while the structural vibrations around $8 \mathrm{~Hz}$ are filtered out.

After applying the appropriate filtering to the balance signals the number of oscillation cycles to achieve a good mean cycle was examined. In this experiment the effect of the number of cycles for the runs and tares was examined to see how it affected the data integrity and standard deviation of the balance moment for a given cycle relative to the mean cycle. Figures 12 and 13 show how the standard deviation changes as the number of cycles is increased for the tare and run, respectively. It can be seen that the standard deviation is very small on the tare run and that adding more cycles has little effect while for the wind-on run there is a notable difference between 20 and 40 cycles, but going to 80 cycles does not significantly improve the standard deviation. During testing the tare runs were made with 20 cycles and the wind-on data were taken with 40 cycles. 
The roll damping derivate results will be discussed first followed by the yaw damping. Figure 14 shows how the experimental $\bar{c}_{l_{p}}$ data compares with the analytical as a function of $\alpha$. The analytical data shown in this paper were calculated using DATCOM. The data shows that the two methods compare very well for $\bar{c}_{l_{p}}$ through $\alpha \approx 5^{\circ}$, which

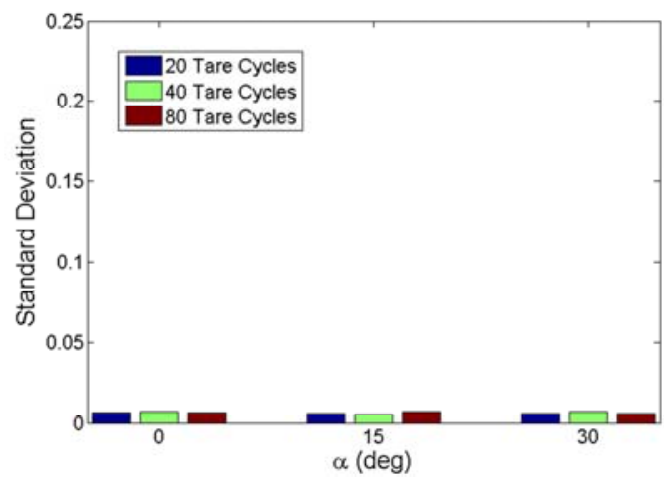

Figure 12. Effect of number of cycles on tare standard deviation for $\alpha=0^{\circ}, 15^{\circ}$, and $30^{\circ}$.

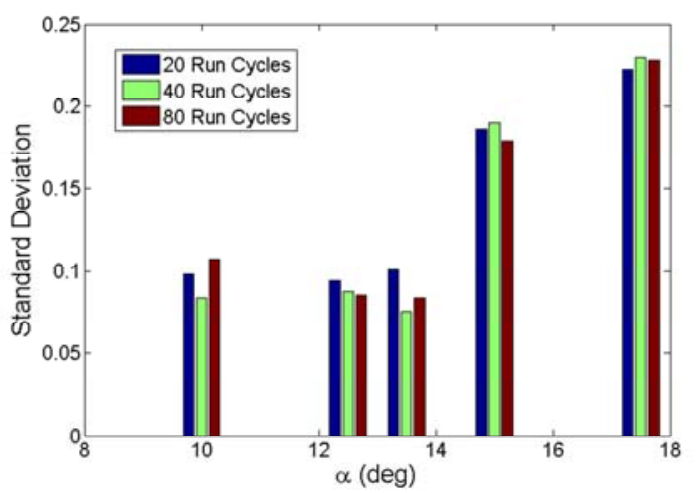

Figure 13. Effect of number of cycle on wind-on run standard deviation for various $\alpha$ 's.

covers the nominal trim range of the airplane. The experimental data shows that $\bar{c}_{n_{p}}$ varies in the linear aerodynamic regime, which is not predicted by the analytical method. This is likely due to the wing influencing the flow field over the vertical stabilizer as the angle of attack increases which results in the negative slope up to stall.

Next plots were made to examine the effect of reduced frequency and rate on the damping derivatives. To begin, the hysteresis loops of the total rolling moment as measured by the balance were examined at $0.53 \mathrm{~Hz}$ from $5^{\circ}$ amplitude to $22^{\circ}$ (Fig. 15). The black lines trace each oscillation, while the red line is an average of all of the oscillations cycles (mean cycle). It can be seen that the loops are angled diagonally from top-left to bottom-right which is a sign of static stability. An indicator of dynamic stability is the fact that the direction of rotation (arrows in plot) around the loops is counter-clockwise. Figure 16 shows the corresponding values of $\bar{c}_{l_{p}}$ versus $\alpha$ at a constant reduced frequency. The data shows only a small dependence of $\bar{c}_{l_{p}}$ on $\hat{p}$ up to $\alpha=20^{\circ}$. There is a noticeable effect between $\alpha=30^{\circ}$ and $40^{\circ}$, most likely because of the non-linear flow effects over the wing at these
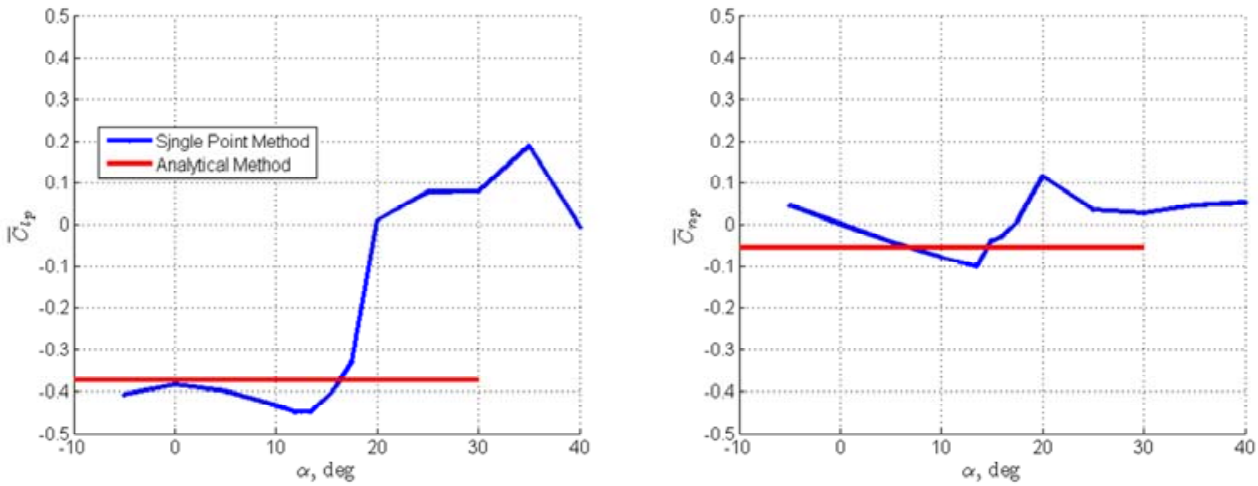

Figure 14. Comparison of single point method to analytical prediction

high angles of attack. Also since a negative $\bar{c}_{l_{p}}$ indicates stability one can see that FASER has stable roll damping through stall, with almost constant values of $\bar{l}_{l_{p}}$ up to $\alpha=15^{\circ}$. Between $\alpha=15^{\circ}$ and $20^{\circ}$ stable roll damping decreases until it becomes neutrally stable or unstable (based on rate) up to $\alpha=40^{\circ}$. 
Holding the non-dimensional rate constant while changing the frequency produces the hysteresis loops seen in Fig 17. To hold $\hat{p}$ constant while changing the frequency the amplitude must decrease as frequency is increased. Extraction of the roll damping derivative from these hysteresis loops over the range of angle of attack is shown in

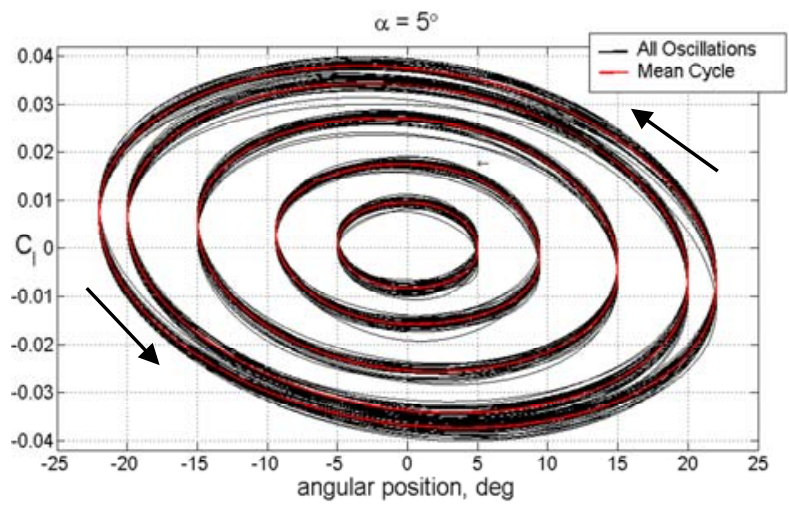

Figure 15. Hysteresis loops of $0.53 \mathrm{~Hz}$ oscillations with amplitude from 5 to 22 degrees at $\alpha=5^{\circ}$.

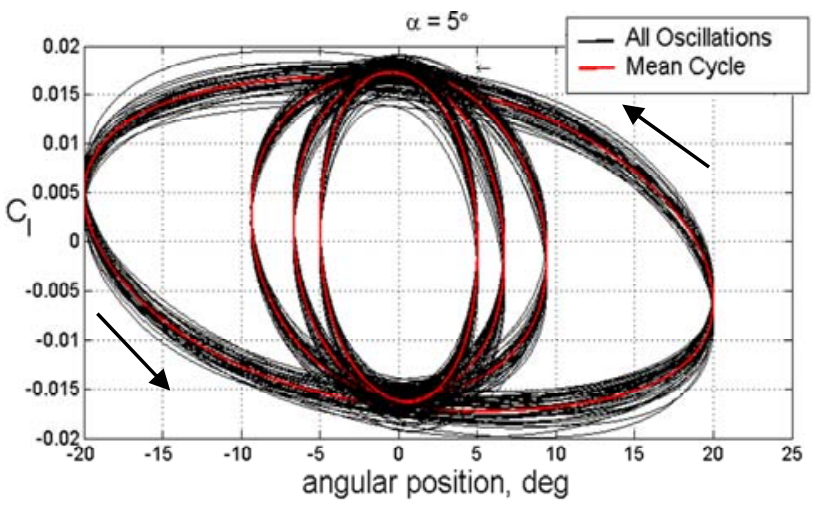

Figure 17. Hysteresis loops at $\hat{p}=0.042$ from $0.25 \mathrm{~Hz}$ to $1 \mathrm{~Hz}$ with $\alpha=5^{\circ}$

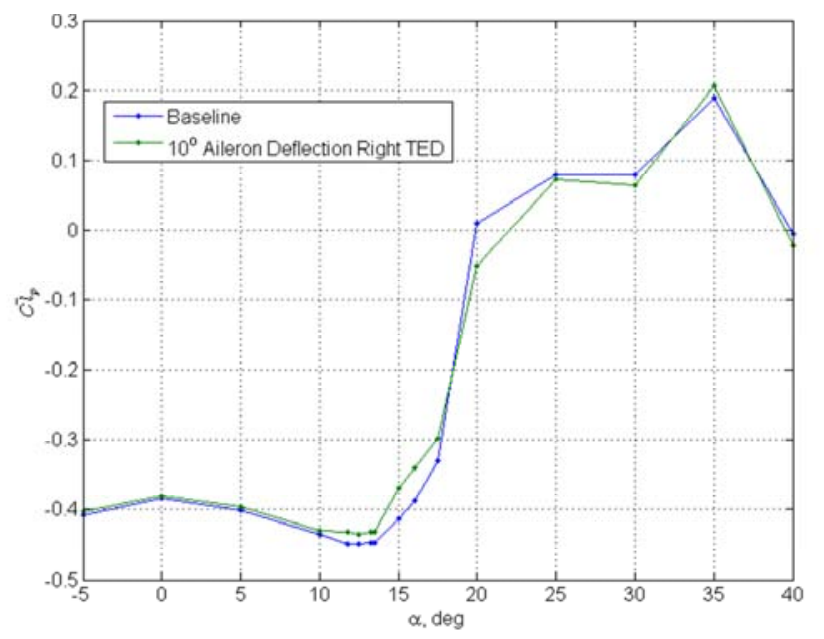

Figure 19. Effect of aileron deflection on roll damping coefficient

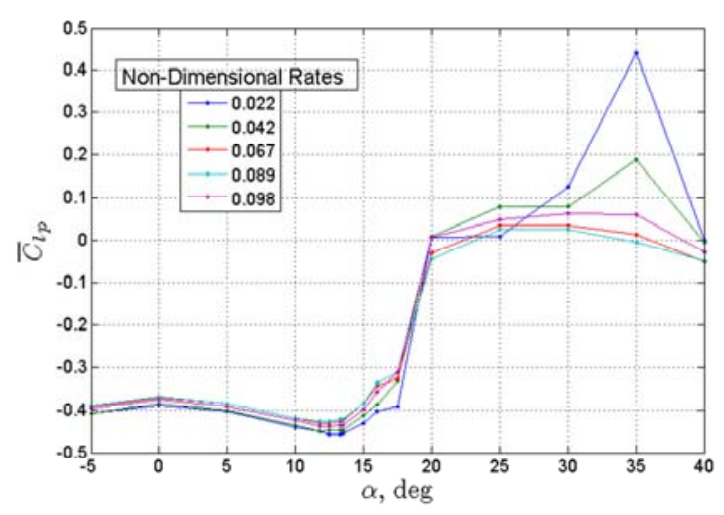

Figure 16. Roll damping coefficients vs. $\alpha$ with reduced frequency $k=0.255$.

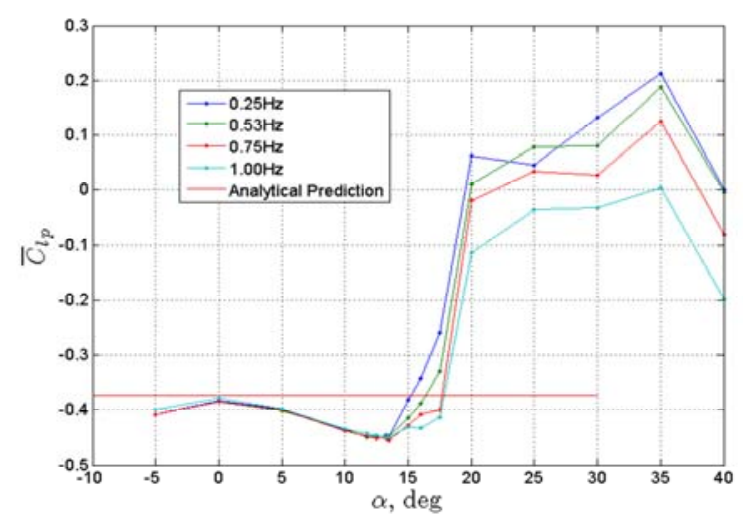

Figure 18. Effect of $k$ on roll damping and compared to the analytical value. $\widehat{p}=0.042$.

Fig 18. It can be noted that $\bar{c}_{l}$ has only a small dependence on $k$ for $\alpha<15^{\circ}$ and also that the analytical value used in the simulation makes an adequate prediction for the lower angles of attack.

The effect of aileron deflection on roll damping was examined (Fig. 19). A deflection of $10^{\circ}$ was made with the right wing aileron set trailing edge down. The data shows that the aileron deflection has very little effect on the roll damping. Therefore, this reduces the complexity of the aerodynamic model for roll damping since aileron deflections effects do not have to be modeled. 
The analysis of yaw forced oscillation test data began by looking at how the experimental data compared to the analytical prediction (Fig. 20) The analytical prediction match the forced oscillation results for $\bar{c}_{n_{r}}$ up to stall angle of attack showing that analytical values are good over a large $\alpha$ range. The agreement between the two data sources over such a large $\alpha$-range is probably due to the very conventional wing planform. Next, the effect of reduced frequency parameter was examined and is shown in Fig. 21. This plot shows $\bar{c}_{n_{r}}$ vs. $\alpha$ with $\hat{r}=0.067$ and it can be seen that there is a fair dependence on frequency near stall $\left(\alpha \approx 15^{\circ}\right)$ where higher

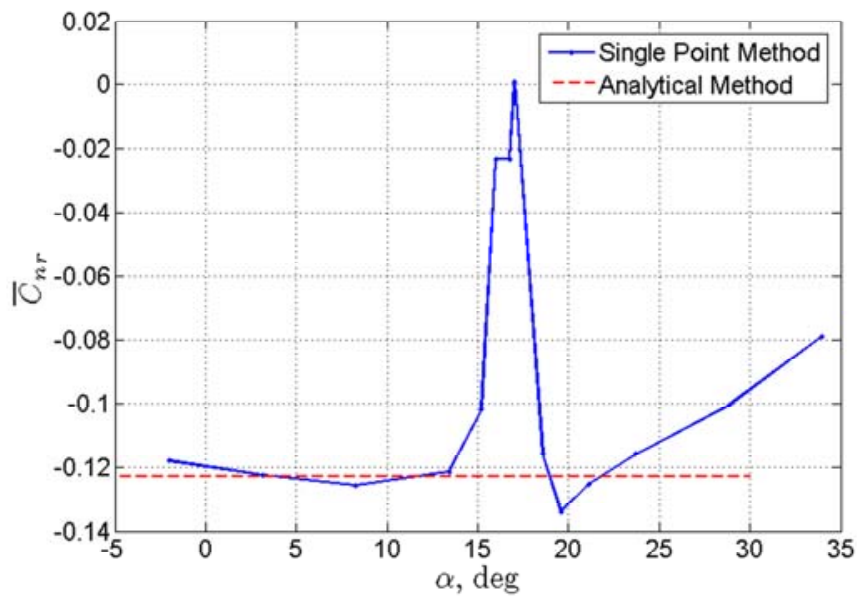

Figure 20. Yaw damping derivatives comparing forced frequencies yield more yaw damping stability. Putting this frequency dependence into the simulation requires a more general model structure than commonly used. One approach using indicial models can be found in Ref. 9. Also shown is the analytical value and it does a good job of approximating the forced oscillation results under $\alpha=5^{\circ}$ and fortuitously near $\alpha=20^{\circ}$. This frequency effect near stall might be caused by the wing wake separated flow impinging the vertical tail at a frequency out-of-phase from the oscillation frequency. Figure 22 shows the effect of non-dimensional rate on yaw damping while holding $k$ constant at 0.12 . This plot shows that there is much less dependence of $\bar{c}_{n_{r}}$ on $\hat{r}$ than there is on $k$, but some dependence still exists near stall.

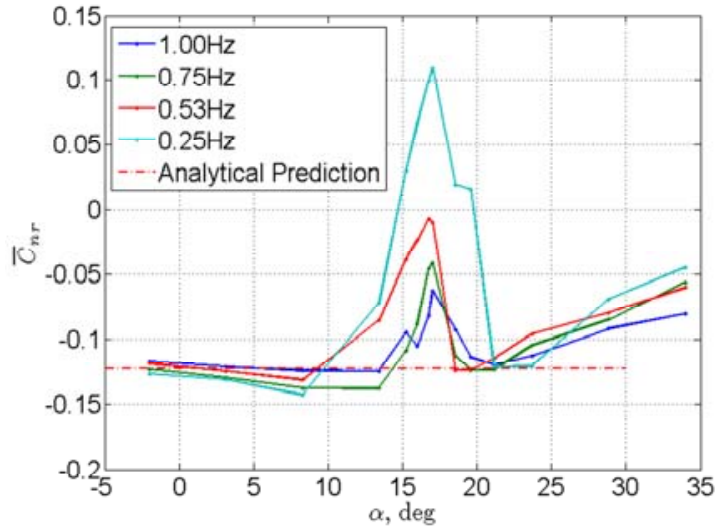

Figure 21. Effect of reduced frequency on the yaw damping derivative for $\hat{\boldsymbol{r}}=0.067$.

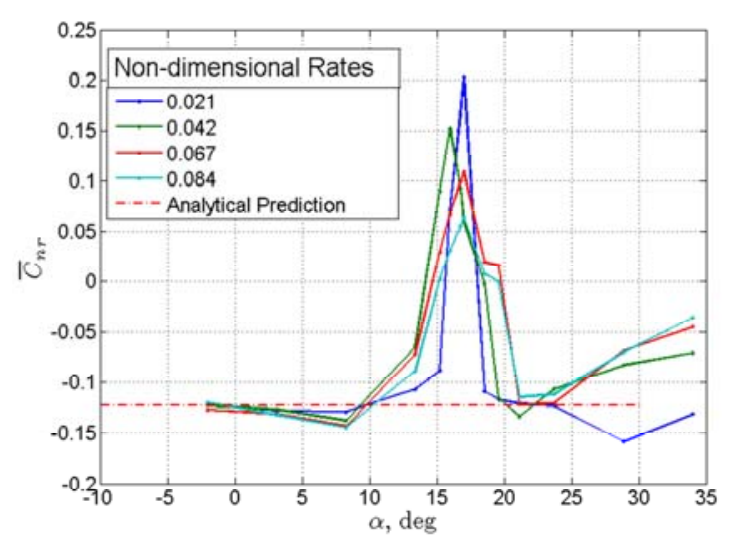

Figure 22. Effect of $\hat{\boldsymbol{r}}$ on the yaw damping derivative for $k=0.12$.

\section{Conclusion}

In the NASA LaRC 12-FT Low-Speed Tunnel, the roll and yaw damping derivatives were experimentally determined for FASER, an unmanned aerial vehicle. FASER is used at NASA LaRC as a generic, low-cost, easyto-fly UAV platform for flight dynamics research. The damping derivatives were measured using the forced oscillation technique. Static data were also measured as a means to assure that the current wind tunnel setup did not contain any anomalies that should be addressed before proceeding to the more complex forced oscillation technique. The results from the forced oscillation measurements showed that:

1. The number of cycles required to obtain an adequate mean cycle for the wind-off tare was 20 and for the wind-on run was 40 . 
2. $\bar{c}_{l_{p}}$ and $\bar{c}_{n_{r}}$ show very good agreement with analytical values in the linear pre-stall aerodynamic regime. Experimentally derived damping values differ significantly from analytical predictions at stall and post-stall angles of attack.

3. The airframe is dynamically stable in roll for about 5 degrees above stall.

4. $\quad \bar{c}_{l_{p}}$ shows a dependency on non-dimensional roll rate and reduced frequency parameter above stall angle of attack

5. The airframe is dynamically stable in yaw below the stall angle of attack with a near constant value of $\bar{c}_{n_{r}}$ that agrees very well with analytical predictions.

6. From $\alpha \approx 10^{\circ}$ to $20^{\circ}$ yaw damping shows a very strong dependence on reduced frequency parameter.

Future work will include replacing the analytical values currently in the simulation aero database with the nonlinear experimental data. The data table will be a function of angle of attack and non-dimensional rate to improve the capability of modeling the aircraft characteristics near stall. A comparison of the simulation flight characteristic using the analytically derived values against the experimental values would show how useful this type of testing is and how important it is to collect forced oscillation data for this type of UAV. Although this experiment could not gather pitch data because of physical hardware limitations, there is a plan to obtain the pitch damping derivatives on a newly developed forced oscillation rig in the NASA 20ft Vertical Spin Tunnel (VST). Also, future plans call for conducting a rotary balance test in the VST to obtain the rotary derivatives so that simulation studies can be conducted of spin and spin recovery type maneuvers.

\section{Acknowledgments}

The authors would like to thank the staff members in the NASA LaRC Flight Dynamics Branch who were very helpful and supportive in conducting the wind tunnel test. The authors would also like to thank Austin Murch, University of Minnesota, for providing valuable insight during the test planning phase as well as flight test data. The authors want to acknowledge Gene Adams (NASA LaRC) for his assistance preparing the model for testing. Lastly, this work was conducted as a part of the NASA LARSS internship program.

\section{References}

${ }^{1}$ Morelli, E.A., and DeLoach, R., "Response Surface Modeling using Multivariate Orthogonal Functions," AIAA 2001-0168, $39^{\text {th }}$ AIAA Aerospace Sciences Meeting and Exhibit, January 2001.

${ }^{2}$ Favaregh, N., "Global Modeling of Pitch Damping from Flight Data," Master's Thesis, Old Dominion University, May 2006.

${ }^{3}$ Monzon, B.R., "Nonlinear Simulation Development for a Sub-Scale Research Airplane," GWU/JIAFS Masters Thesis, August 2001

${ }^{4}$ Garza, F.R. and Morelli, E.A., “A Collection of Nonlinear Aircraft Simulations in MATLAB ${ }^{\circledR}$,” NASA TM-2003-212145, January 2003.

${ }^{5}$ Owens, D. B., Cox, D.E., and Morelli E.A., "Development of a Low-Cost Sub-Scale Aircraft for Flight Research: The FASER Project," AIAA 2006-3306, 25 ${ }^{\text {th }}$ AIAA Aerodynamic Measurement Technology and Ground Testing Conference, June 2006.

${ }^{6}$ Owens, D.B., Brandon, J.M., Croom, M.A., Fremaux, M., Heim, E., and Vicroy, D.D., “Overview of Dynamic Test Techniques for Flight Dynamics Research at NASA LaRC," AIAA 2006-3146, 25 ${ }^{\text {th }}$ AIAA Aerodynamic Measurement Technology and Ground Testing Conference, June 2006.

${ }^{7}$ Chambers, J.R. and Grafton, S.B., "Static and Dynamic Longitudinal Stability Derivatives of a Powered 1/9-Scale Model of a Tilt-Wing V/STOL Transport,” NASA TN D-3591, September 1966.

${ }^{8}$ Klein, Vladislav, Murphy, Patrick C., and Szyba, Nathan M., "Analysis of Wind Tunnel Lateral Oscillatory Data of the F16XL Aircraft,”NASA/TM-2004-213246, 2004.

${ }^{9}$ Murphy, P.C., Klein, V., "Transport Aircraft System Identification Using Roll and Yaw Oscillatory Wind Tunnel Data," AIAA 2010-8122, August, 2010. 